\title{
2-D to 3-D Refinement of Post Mortem Optical and MRI Co-registration
}

\author{
C. Kenwright ${ }^{1}$, É. Bardinet ${ }^{2}$, S.A. Hojjat ${ }^{1}$, G. Malandain ${ }^{2}$, N. Ayache ${ }^{2}$, and \\ A.C.F. Colchester ${ }^{1}$ \\ 1 University of Kent,Canterbury, Kent, CT2 7NZ \\ \{c.kenwright, a.colchester, s.a.hojjatoleslami\}@kent.ac.uk \\ 2 EPIDAURE Research Project, \\ INRIA, 2004 route des Lucioles BP 93, \\ 06902 SOPHIA ANTIPOLIS Cedex - FRANCE \\ \{Eric.Bardinet, Gregoire.Malandain, Nicholas.Ayache\}@sophia.inria.fr
}

\begin{abstract}
Accurate co-registration of MR images of the brain with pathological data from the same patient is required for many applications, including validation of in-vivo MRI processing. We present preliminary results from a 2-D optical to $3-\mathrm{D}$ MRI registration process, which depends on initialisation using an initial 3-D reconstruction of post-mortem whole-brain or hemisphere slices from independent $2 \mathrm{D}$ images followed by 3 -D to 3 -D registration. Our overall approach progressively models the acquisition process, and the final stages account for variations in slice thickness and other errors that can occur as a result of the specimen preparation and earlier processing. Our method can be used in most neuropathology laboratories where thick slices are cut. Alternative approaches depend on sectioning by macrocryotomes, which are not generally available and have limited applicability in conditions such as CJD where infectivity risks are high.
\end{abstract}

\section{Introduction}

Accurate co-registration of MR images of the brain with pathological data from the same patient is required for many applications, including validation of in-vivo MRI processing. Our application is concerned with the investigation of the relationship between localised intensity abnormalities in brain MRI of patients with Creutzfeldt-Jakob disease (CJD) and underlying pathological changes. The reasons for these regional variations are not well understood but they are of great importance in differential diagnosis (e.g. discrimination between sporadic and new variant CJD [6]). Accurate mapping between MRI and post mortem pathology measures will allow clearer understanding of the causes of MRI changes, the discovery of abnormalities in one data source (e.g. histology) to focus attention on specific regions of interest in the other data source (e.g. MRI) and the tracking of the 3-D distribution of areas that have been sampled and studied in pathological studies of distributed processes. 
Accurate mapping between MRI and post mortem pathology measures will allow clearer understanding of the causes of MRI changes. It will make possible validation of computer analysis of MR images (e.g. tract tracing in diffusion tensor images) by detailed histological studies. It will allow discovery of abnormalities in one data source (e.g. histology) to focus attention on specific regions of interest in the other data source (e.g. MRI) potentially revealing changes that would not otherwise be detected. It is difficult in the pathological studies of widely distributed processes to keep track of the 3-D distribution of areas that have been sampled and studied which mapping between MRI and pathology will greatly improve.

Accurate mapping between histology and human MRI depends on the acquisition of macroscopic photographs of large tissue slices. These optical images need to bridge the resolution gap between MRI and microscopy, so that any microscopic sample can be identified within the optical dataset and co-registration can be performed with MR data. In order to register the optical data with the MR, other work 2/385 has used the 2-D serial data to create an "optical volume", acquired with a micro- or macrotome and with a reasonably low (submillimetre) slice thickness. If the serial sections are taken carefully with a fixed camera position, serial section photographs of the cut surface of the large brain block, still mounted in the macrotome, can be acquired in a common coordinate frame 8, or artificial fiducials can be used, whether internal (placed within the tissue sample) [5] or external (outside the tissue sample) 2] to provide a registration basis. The selection of $2-\mathrm{D}$ registration criteria is important as individual sections are subject to independent geometric distortions and this can lead to an inaccuracies in the reconstructed 3-D volume if distortions are propagated. This accurate registration allows the creation of a "reference volume" 7]. The reference volume can be co-registered with the MR [2] or used solely to localise individual histological sections [7] by virtue of the fact that adjacent thin sections can be considered at the same position. Where it is not possible to create such a reference volume attempts have been made to combine the reconstruction and alignment in an iterative process 12 . It is also possible to align the sections through the careful arrangement of the tissue before sectioning, using corresponding landmarks in the tissue and MR for orientation, so that sections are taken already aligned with the MR [13].

Groups working on human brain have often used a macrocryotome to acquire large field-of-view thin slices. However macrocryotomes are only available in a small number of laboratories and their use would not allow prompt neuropathology examinations which may be essential for clinical or medico-legal reasons. Furthermore the transmissible and highly resistant nature of the infectious prion protein in CJD means that complex precautions are necessary in the handling of CJD brains. The approach presented here is to allow registration of optical to MRI data performed with minimal deviation from normal pathological procedures. During preparation of the brain for microscopic examination, the pathologist cut the brain with a hand-held knife resting against a guide, into coronal slices of chosen thickness between 7 and $15 \mathrm{~mm}$. Each slice was then 
placed in a specially designed frame, or jig [4], with transparent faces. On each side of the jig, fiducials were precisely engineered to lie exactly opposite each other. Both the posterior and the anterior face of each slice were photographed with a digital camera at high resolution by turning the jig over.

The registration is performed using a seven stage algorithm, summarised in Table 1 The first and second stages are between (inter-) and within (intra-) slice registration using an iterative closest point (ICP) algorithm to align fiducials and an intensity based measure to align the image of the anterior face of one slice with the posterior face of the next. The third stage combines the matrices for all within and between slice transformations and the slice thickness, so that the entire series of images was put in a common coordinate frame, creating an optical volume [4. Stage 4, as performed in [1] was to register this optical volume with the MR of the same brain. Stages 1-4 were performed in the current work with slight differences compared to 4,1. Stage 4 is explained in the Methods section.

The registration of the optical volume with MRI, is complicated by the physical features of the tissue. Significant differences between the in vivo and post mortem brains - mainly due to shrinkage and processing post mortem brain makes the matching very difficult. However, distortion caused by cutting the brain, in addition to other problems with the photographs of brain slices such as non-cut surface (parts of the outer brain surface which are oblique and visible en face), can lead to inaccuracies in the registration process between slices and therefore inaccuracies as a representation of the volumetric brain. The optical volume assumes that all slices have parallel faces and are of constant thickness in reality slices may be of slightly uneven thickness and there may be systematic deviations across the slice (i.e. the slices may be slightly wedge shaped).

Previous work 4,1] performed reconstruction and co-registration using globally linear parametric measures. Here the aim is to obtain a reasonable 3-D representation using the optical volume as an initialisation stage, while introducing more sophisticated non-rigid updating applied to each slice independently using the MR volume as the model for controlling the process (Stages 4 to 7) in order to recover the deformations inherent in the acquisition process. In the present paper the earlier stages are restricted to rigid transformations. The new approach is to use iterative refinement of the optical volume through a sequence of global 3-D registrations and local 2-D registrations. This is coupled with a gradual increase in the number of degrees of freedom as the search space reduces on approaching anatomical correspondence. The refinement culminates in a 2-D optical to 3-D MRI step.

\section{Materials and Methods}

Seven subjects were studied, with both post mortem optical imaging (using our protocol) and MR imaging (in vivo and/or post mortem). Three subjects had both in vivo and post mortem MR. The reconstructed optical volumes were orientated so that the slices were positioned in the $x$ and $y$ planes (a coronal slice). In some brains only one cerebral hemisphere was available for slicing, while 
in others a complete coronal slice was generated. Seven stages were identified in the overall registration process, of which stages 1-3 have been described above. Stages 5-7 are the refinement steps described in the present paper. Stages 5 (3-D intensity based registration) and 6 (2-D planar registration) are iterated with the reconstruction of the optical volume, with freedom of movement increasing with iterations. Rigidity constraints were relaxed further in stage 7 to allow movement in new directions.

Table 1. Summary of all 7 stages in the process of Optical to MR registration. PMOP = Post Mortem OPtical data. Relaxing = initially rigid but increasing degrees of freedom with iterations. Smoothed $=$ transformation smoothing applied. In-plane $=$ movement allowed only in $x y$ plane of volume. Out-of-plane $=$ movement allowed in the $z$ direction as well as $x$ and $y$. For stages 4-7 the first dataset is "floating", the second the "reference" or "target"

\begin{tabular}{|l|l|l|l|l|l|}
\hline Datasets & Objects & Constraints & $\begin{array}{l}\text { Cost } \\
\text { Function }\end{array}$ & Optimiser \\
\hline 1 & $\begin{array}{l}\text { Antcrior and postcrior } \\
\text { faces of same brain slice } \\
\text { (within-slice registration) }\end{array}$ & Jig Fiducials & Rigid & $\begin{array}{l}\text { Distance } \\
\text { Metric }\end{array}$ & $\begin{array}{l}\text { Hicrarchical, } \\
\text { Iterative }\end{array}$ \\
\hline 2 & $\begin{array}{l}\text { Anterior and posterior } \\
\text { faces across a cut } \\
\text { (between-slice } \\
\text { registration) }\end{array}$ & $\begin{array}{l}\text { Pixel } \\
\text { Intensities }\end{array}$ & Rigid & $\begin{array}{l}\text { Correlation } \\
\text { Coefficient }\end{array}$ & $\begin{array}{l}\text { Hierarchical, } \\
\text { Block } \\
\text { Matching }\end{array}$ \\
\hline 3 & $\begin{array}{l}\text { Reconstruction of } \\
\text { Optical volume }\end{array}$ & $\begin{array}{l}\text { Image } \\
\text { Coordinates }\end{array}$ & Rigid & Not Applicable & $\begin{array}{l}\text { Matrix } \\
\text { Multiplication }\end{array}$ \\
\hline 4 & $\begin{array}{l}\text { 3D PMOP surface to } \\
\text { 3D MR surface }\end{array}$ & $\begin{array}{l}\text { Outer Brain } \\
\text { Surface }\end{array}$ & Rigid & $\begin{array}{l}\text { Distance } \\
\text { Metric }\end{array}$ & $\begin{array}{l}\text { Hierarchical, } \\
\text { Iterative }\end{array}$ \\
\hline 5 & $\begin{array}{l}\text { 3D PMOP volume to } \\
\text { Voxel } \\
\text { Intensities } \\
\text { 3D MR volume }\end{array}$ & $\begin{array}{l}\text { Rigid to } \\
\text { Affine } \\
\text { (relaxing) }\end{array}$ & $\begin{array}{l}\text { Correlation } \\
\text { Ratio }\end{array}$ & $\begin{array}{l}\text { Gradient } \\
\text { Descent }\end{array}$ \\
\hline 6 & $\begin{array}{l}\text { 2D PMOP slice to } \\
\text { 2D MR slice } \\
\text { (in-plane) }\end{array}$ & $\begin{array}{l}\text { Voxel } \\
\text { Intensities }\end{array}$ & $\begin{array}{l}\text { Rigid } \\
\text { (smoothed) }\end{array}$ & $\begin{array}{l}\text { Correlation } \\
\text { Cocfficient }\end{array}$ & $\begin{array}{l}\text { Hierarchical } \\
\text { Block } \\
\text { Matching }\end{array}$ \\
\hline $\begin{array}{l}7 \\
\text { 2D PMOP slice to } \\
\text { 3D MR volume } \\
\text { (out-of-plane) }\end{array}$ & $\begin{array}{l}\text { Voxel } \\
\text { Intensities }\end{array}$ & Rigid & $\begin{array}{l}\text { Correlation } \\
\text { Ratio }\end{array}$ & $\begin{array}{l}\text { Gradicnt } \\
\text { Descent }\end{array}$ \\
\hline
\end{tabular}

\subsection{Initialisation Using ICP: Stage 4}

The outer brain surface in the MR and optical volumes were extracted using a Canny based edge detector [9]. The extracted point sets were then registered without deformation with an ICP, constraining the transformation to rigid motion only. There were inherent inaccuracies in the process as the extracted surface from the optical volume does not always represent the actual outer brain surface. 
This is in part due to the detection of the non-cut surface of the brain rather than the actual cortex boundary. In the case of in vivo MR the outer surface often differed from that in the post mortem optical volume as the post mortem brain was removed by the pathologist from the head. As a result it was necessary to estimate in the in vivo MR where the pathologist had cut the brain stem and cerebellum. It did however provide an adequate starting point for the later stages of the registration process.

\subsection{D Intensity Based Registration: Stage 5}

The optical and MR volumes were registered in 3-D, intially using rigid constraints, using an intensity based measure, which was a version of the correlation ratio [1],

$$
\eta(I \mid J)=1-\frac{\operatorname{Var}(I-\mathrm{E}(I \mid J))}{\operatorname{Var}(I)},
$$

for voxel sets $I$ and $J$, where $\mathrm{E}(I \mid J)$ represents the conditional expectation of $I$ in terms of $J$. This optimises the registration over the whole brain volume.

\subsection{D Intensity Based Registration: Stage 6}

Following the 3-D registration of the volumes, the corresponding coronal slice from the MR for each slice of the optical volume was extracted and the resulting images were registered in 2-D. The transformation matrices over all slices were smoothed using a scheme based on the difference between residuals for consecutive slices, each slice exerting an influence over others.

$$
\begin{gathered}
\mathrm{r}_{i j}=\mathrm{T}_{i}^{-1} \mathrm{~T}_{j}, \\
\mathrm{r}_{i}=\sum_{j} \psi(j) \mathrm{r}_{i j}, \\
\mathrm{~T}_{i}=\mathrm{T}_{i} \mathrm{r}_{i},
\end{gathered}
$$

where $\mathrm{T}_{i}$ is the transformation for slice $i$ and $\psi(j)$ represents a Gaussianweighted regularisation function. This step was based on the idea that an important group of distortions are 3-D processes, e.g. squashing the brain from one side, which would therefore vary smoothly between slices. An exceptional transformation could arise from either a poor registration here or a slice that was not well aligned during the optical volume construction. In this case repeated iterations brought the slice into alignment while at the same time avoiding potentially irrecoverable registration errors in the global registration. Initially this stage was restricted to a rigid transformation. The cost function used was the correlation coefficient,

$$
\rho(I, J)=\frac{\operatorname{Cov}(I, J)}{\sqrt{\operatorname{Var}(I)} \sqrt{\operatorname{Var}(J)}},
$$

where $I$ and $J$ represent image sets used with a block matching algorithm [10], which assumes only two tissue types per block and therefore a linear intensity relationship is modelled. 


\subsection{Iteration and Relaxation of Degrees of Freedom: Stage 5 and 6}

Following the reconstruction of the optical volume, it was then re-registered with the MR volume. The constraints, if a rigid registration returned the identity, were relaxed, to anisotropic scaling (similarity) and then affine in subsequent iterations. The corresponding optical and MR slices were then extracted again and registered in 2-D. The optical volume was then reconstructed using the unchanged $z$ coordinate.

\subsection{Final Relaxation of Rigidity Constraints with 2-D Optical to 3-D MR Co-registration: Stage 7}

All individual slice movement so far allowed has been in the $x y$ plane of the $2-\mathrm{D}$ slices or globally in 3 -D. This was performed using a $3-\mathrm{D}$ registration of a single optical slice to the local neighbourhood of the corresponding slice in MR volume. The correlation ratio was again used as the cost function. The local neigbourhood was approximately the thickness of one optical slice in the $z$ axis (and the full $x y$ plane as used in stage 6) centered around the optical slice $z$ coordinate.

\section{Results}

Registration was performed on seven sets of optical and MR volumes up to the end of stage 6 . Two optical volumes have so far been used for the final 2-D to 3 -D refinement in stage 7 . The results were assesed by examining features on the cortical boundary and deep in the cerebrum and assesing anatomical correspondence, using visual inspection of image overlays, subtraction images and linked cursors.
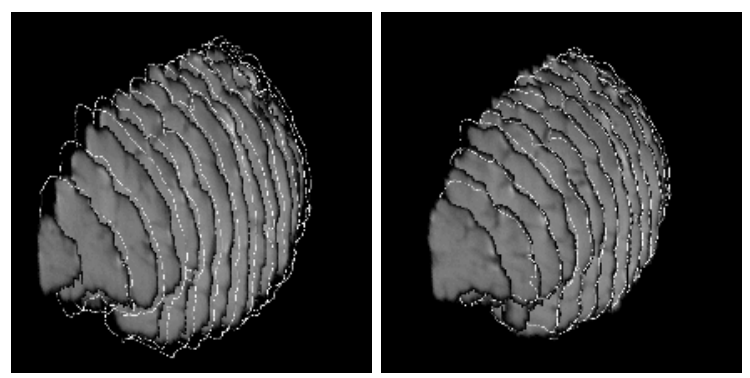

Fig. 1. 3-D views of the extracted slices from a brain hemisphere following the registration process. The MR is shown in dark grey and the optical volume is in outline. The left image has been registered using an iterative closest point algorithm only and the right image has been registered up to stage 7 . Movement and scaling transformations have been applied to the MR volume for visualization. 
Generally, an excellent correspondence was achieved between the volumes. A 3-D view of a brain hemisphere is shown in Figure 1 which shows the correspondence of the slices over the whole volume. Registered slices of a whole brain are shown in Figure 2 where a clear improvement can be seen on the superior medial edge of the cortex. The correction of distortions introduced during the construction of the optical volume is shown in Figure 3 For comparison this is presented with the results from previous work [1 where a single 3-D affine transformation was used without independent slice movement.

The effect of the final 2-D to 3-D co-registration was further evaluated by analysis of the out-of-plane and in-plane transformations. The out-of-plane rotations were calculated from the intersection of the resulting planes after rotation about the $x$ and $y$ planes. The in-plane movement is the magnitude of the $x y$ translation vector (Table 2). For three optical slices the registration failed. The calculated transformations were physically impossible with translations of $64.8 \mathrm{~mm}$ to $33.9 \mathrm{~mm}$ and rotations of $54.2^{\circ}$ to $30.1^{\circ}$. Good correspondence of feature was observed for all the other images; these results are shown in Table 2.
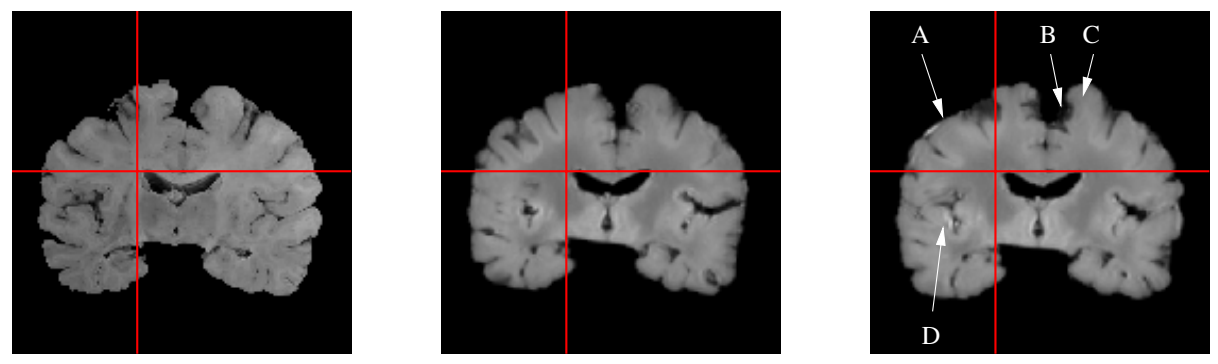

Fig. 2. Corresponding slices of whole brain in the optical (left) and post mortem brains. Left: the original optical slice; centre: the corresponding MR slice following registration using the ICP algorithm (stage 4); right: MR following the iteration of independent in-plane and 3-D global registration (stages 5 and 6 ). The cursors mark the same point in each image. A: improved matching of gyri. B: non-cut surface in optical image is (correctly) ignored bringing the true boundary correctly into alignment at C. D: deep sulci are correctly matched.

\section{Discussion}

It has been shown that a good correspondence between the post mortem optical data and MRI of the same patient can be achieved with the minimum possible interference with standard neuropathology procedures. The quality of results was judged by visual checking of correspondence of recognised features and by assessing the consistency of quantitative data about out-of-plane co-registration results with knowledge of inaccuracies arising during cutting of slices. 

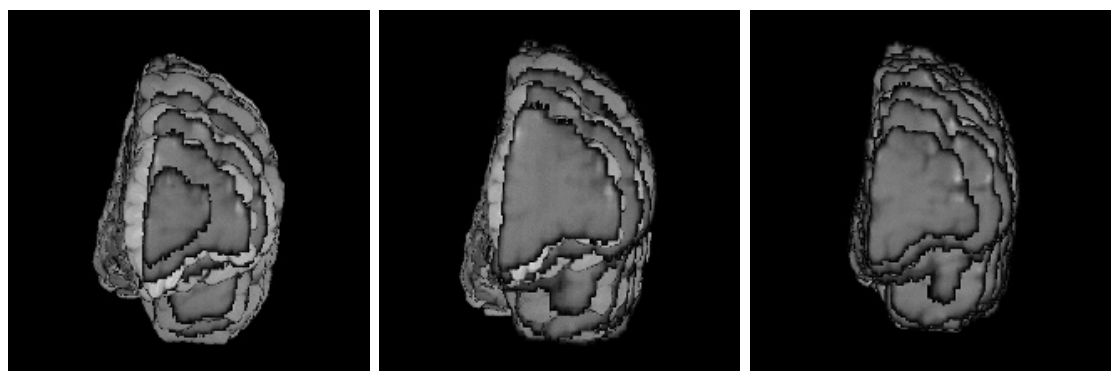

Fig. 3. View from front of 3-D brain images illustrating the removal of skew during progressive refinement of registration. Left: images registered with ICP algorithm only (Stage 4). Centre: images registered using an affine intensity based algorithm (affine version of Stage 5 only). Right: images registered using iterated in-plane registration and rigid intensity based algorithm (Stages 5 and 6 )

Table 2. Quantitative measurements of independent out-of-plane slice movement effected by stage 7 . The transformations are decomposed into in-plane components, representing similar transformations to those seen in stage 6 , and out-of-plane components, which represent the new degrees of freedom allowed in stage 7

\begin{tabular}{|c|c|c|c|c|}
\hline Direction & Movement & $\begin{array}{c}\text { Mean } \\
\text { Magnitude }\end{array}$ & Max & Min \\
\hline In-plane & Translation $(\mathrm{mm})$ & 0.31 & 4.14 & 0 \\
& Rotation $\left(^{\circ}\right)$ & 0.11 & 2.1 & 0 \\
\hline Out-of-plane & Translation $(\mathrm{mm})$ & 0.18 & 11.3 & 0 \\
& Rotation $\left(^{\circ}\right)$ & 1.49 & 8.22 & 0 \\
\hline
\end{tabular}

The spatial data were derived from slices about 10mm thick. There are several consequences of this. (a) Unlike many thin-section reconstruction approaches, no assumptions were made about the correspondence between anatomical features on the front and back of the same slice; this is a strength of the presented approach. (b) The images of the cut surface of the slice faces reflect the surface tissue only, unlike MRI data where individual voxel values integrate information through a significant volume. (c) The images of cut surfaces of adjacent slices should thus correspond exactly, without partial volume effects. (d) On the other hand, some of the optical images have the specific disadvantage of showing oblique non-cut surface of slices. Currently these parts of the image are not specifically excluded, but robust registration techniques were relied on that appear successfully to treat these data (from non-cut surface) as outliers which were rejected during optimisation. (e) The wide separation between optical slices made it impossible to validate 3 -D registration using point landmarks. The overall approach has been based on the joint development of image acquisition and post-processing procedures. The registration processes are optimised to correct specific distortions that are known to occur. Several distortions are relatively 
smooth 3-D processes, such as shrinkage. Consistent with this, it was found that the best results from stage 6 were obtained by smoothing the transformations across adjacent slices. On the other hand, it is known that there are also distortions that can affect slices individually. Slice thickness may vary between slices, according to the pressure applied to the brain (to hold it against the cutting table) while slices are cut. More importantly, uneven pressure may cause varying thickness across one slice, particularly if one side of the brain loses contact with the cutting base. These distortions appeared to be well-retrieved by our final $2-\mathrm{D}$ to $3-\mathrm{D}$ registration stage. On average slices needed to be tilted by $1.49^{\circ}$ (out-of-plane), indicating that slice thickness varied by about $2.5 \mathrm{~mm}$ across the slices on average. Evaluation of results obtained so far showed that registration refinement was successful for $90 \%$ of slices. Three failures in stage 7 refinement occurred in which large physically impossible out-of-plane translations and rotations occurred. These were easy to identify and could be suppressed automatically. However we expect that further improvements to stage 7 processing will reduce the incidence of the problem.

One significant slice-specific distortion which is not currently modelled is the independent movement of fragments which are detached from the main body of the slice. However, our approach does successfully co-register the main parts of the slices, reflecting successful rejection of outliers caused by separated fragments.

Acknowledgements. This work was supported in part by the EC-funded projects QAMRIC (BMH4-98-6048) and PVEOUT (2000-00594).

\section{References}

1. E. Bardinet, A. C. F. Colchester, A. Roche, Y. Zhu, S. Ourselin, W. Nailon, S. A. Hojjatoleslami, J. Ironside, S. Al-Sarraj, N. Ayache, and J. Wardlaw. Registration of reconstructed post-mortem optical data with $\mathrm{mr}$ scans of the same patient. In W. J. Niessen and M. A. Viergever, editors, Medical Image Computing and Computer-Assisted Intervention - MICCAI 2001, Lecture Notes in Computer Science 2208, pages 957-965. Springer, 2001.

2. É. Bardinet, S. Ourselin, D. Dormont, G. Malandain, D. Tandé, K. Parain, N. Ayache, and J. Yelnik. Co-registration of histological, optical and $\mathrm{mr}$ data of the human brain. In Takeyoshi Dohi and Ron Kikinis, editors, Medical Image Computing and Computer-Assisted Intervention - MICCAI 2002, Lecture Notes in Computer Science, pages 548-555. Springer, September 2002.

3. U. Bürgel, T. Schormann, A. Schleicher, and K Zilles. Mapping of histologically identified long fiber tracts in human cerebral hemispheres to the mri volume of a reference brain: position and spatial variability of the optic radiation. NeuroImage, 10:489-499, 1999.

4. A. C. F. Colchester, S. Ourselin, Y. Zhu, E. Bardinet, Y. He, A. Roche, S. AlSarraj, W. Nailon, J. Ironside, and N. Ayache. 3-d reconstruction of macroscopic optical brain slice images. In S. Delp, A. M. DiGioia, and B. Jaramaz, editors, Medical Image Computing and Computer-Assisted Intervention - MICCAI 2000, Lecture Notes in Computer Science, pages 95-105. Springer, 2000. 
5. Alberto F. Goldszal, Oleh J. Tretiak, Peter J. Hand, Sanjay Bhasin, and Donald L. McEachron. Three-dimensional deconstruction of activated columns from 2- $\left[{ }^{14} \mathrm{C}\right]$ deoxy-D-glucose data. Neuroimage, 2:9-20, 1995.

6. A. Hojjat, D. Collie, and A.C.F Colchester. The putamen intensity gradient in cjd diagnosis. In Takeyoshi Dohi and Ron Kikinis, editors, Medical Image Computing and Computer-Assisted Intervention - MICCAI 2002, Lecture Notes in Computer Science, pages 524-531. Springer, September 2002.

7. Wolfgang Knabe, Stefan Washausen, Guido Brunnett, and Hans-Jürg Kuhn. Use of 'reference series' to realign histological serial sections for three-dimensional reconstructions of the positions of cellular events in the developing brain. Journal of Neuroscience Methods, 121:169-180, 2002.

8. Micheal S. Mega, Sylvia S. Chen, Paul M. Thompson, Roger P. Woods, Timur P. Karaca, Abhishek Tiwari, Harry V. Vinters, Gary W. Small, and Arthur W. Toga. Mapping histology to metabolism: Coregistration of stained whole-brain sections to premortem pet in alheimer's disease. NeuroImage, 5:147-153, 1997.

9. O. Monga, R. Deriche, G. Malandain, and J.-P. Cocquerez. Recursive filtering and edge tracking: two primary tools for $3 \mathrm{~d}$ edge detection. Image and Vision Computing, 9(4):203-214, August 1991.

10. S. Ourselin, A. Roche, G. Subsol, X. Pennec, and N. Ayache. Reconstructing a $3 \mathrm{~d}$ structure from serial histological sections. Image and Vision Computing, 19(12):25-31, 2001.

11. A. Roche, G. Malandain, X. Pennec, and N. Ayache. The correlation ratio as a new similarity measure for multimodal image registration. In W. M. Wells, A.C.F. Colchester, and S. Delp, editors, Medical Image Computing and Computer-Assisted Intervention - MICCAI 1998, volume 1496 of Lecture Notes in Computer Science, Cambridge, USA, 1998. Springer Verlag.

12. João M. Sanches and Jorge S. Marques. Joint image registration and volume reconstruction for 3d ultrasound. Pattern Recognition Letters, 24:791-800, 2003.

13. J. C. Sørensen, C. R. Bjarkam, E. H. Danielsen, C. Z. Simonsen, and F. A. Geneser. Oriented sectioning of irregular tissue blocks in relation to computerized scanning modalities: Results from domestic pig brain. Journal of Neuroscience Methods, 104:93-98, 2000. 